\title{
Tungsten isotopic evolution during late-stage accretion: constraints on Earth-Moon equilibration
}

\author{
F. Nimmo \\ Department of Earth and Planetary Sciences, University of California Santa Cruz, Santa \\ Cruz CA 95064, USA \\ D.P. O’Brien \\ Planetary Science Institute, Tucson, AZ 85719, USA \\ T. Kleine \\ Institut für Planetologie, Westfälische Wilhelms-Universität Münster, \\ Wilhelm-Klemm-Str. 10, 48149, Münster, Germany
}

\begin{abstract}
We couple the results of N-body simulations of late-stage accretion (O'Brien et al. 2006) to a hafnium-tungsten (Hf-W) isotopic evolution code to investigate the evolution of planetary bodies in the inner solar system. Simulations can simultaneously produce planets having Earth- and Mars-like masses and Hf-W systematics by assuming that the tungsten partition coefficient decreases with increasing semi-major axis (e.g. due to increasing oxidation). Simulations assuming that Jupiter and Saturn occupy circular orbits are more successful at reproducing the Hf-W systematics than those assuming presentday Jupiter and Saturn orbits. To generate Earth-like tungsten anomalies, $30-80 \%$ of each impactor core is required to re-equilibrate with the target mantle. Some model outcomes yield a target and final impactor having similar (Earth- and Moon-like) tungsten anomalies. However, in no case can the inferred lunar Hf/W ratio be simultaneously matched. This result suggests that the Moon isotopically equilibrated with the Earth's mantle in the aftermath of the giant impact (cf. Pahlevan and Stevenson 2007). Alternatively, either the dynamical models which show the Moon being derived primarily from the impactor mantle, or the accretion timescales obtained by the N-body simulations, are incorrect.
\end{abstract}


Keywords:

accretion, tungsten, Moon, impacts

\section{Introduction}

The hafnium-tungsten (Hf-W) isotopic system provides a powerful constraint on the timing of planetary core formation, and thus of planetary accretion (e.g. Jacobsen, 2005; Kleine et al., 2009). Other things being equal, a higher abundance of ${ }^{182} \mathrm{~W}$ in the mantle indicates that accretion finished earlier (see Section 2.2). Measurements of the tungsten isotope systematics of the Earth, Mars, the Moon and asteroids thus allow a chronology of solar system accretion to be established (e.g. Kleine et al., 2009).

Late-stage accretion inevitably involves large collisions (Agnor et al., 1999), which in some cases appear to have resulted in the formation of satellites such as the Moon. The Moon is thought to have formed from the re-accretion of hot material originating from the mantle of a Mars-sized impactor that struck the Earth (e.g. Canup, 2008). From an isotopic point of view, the two key aspects of this story are that: 1) the impactor was differentiated prior to encountering the Earth; and that 2) the bulk of the Moon was derived from impactor mantle material. The first factor is required to explain the apparently low mass of the lunar core and the low Fe content of the Moon: in simulations, the impactor core is rapidly accreted into the target core (Canup and Asphaug, 2001). The second factor implies that the Moon should resemble the composition of the impactor's mantle, not that of the Earth. However, terrestrial and lunar samples display a surprising similarity in isotope compositions of elements that show variations among other solar system bodies. These elements include O (Wiechert et al., 2001), Cr (Lugmair and Shukolyukov, 1998; Trinquier et al., 2008), Ti (Leya et al., 2008) and W (Touboul et al., 2007, 2009). The similarity in O (and Cr and Ti) isotopes could in principle arise if two bodies contained a similar mix of nucleosynthetic and solar nebula components and perhaps formed at similar heliocentric distances (Wiechert et al., 2001), although the probability of such an occurrence is small (Pahlevan and Stevenson, 2007). However, the indistinguishable $\mathrm{W}$ isotopic compositions of the terrestrial and lunar mantles presents more of a problem, because variations in the ${ }^{182} \mathrm{~W} /{ }^{184} \mathrm{~W}$ ratio cannot be produced by varying proportions of different nucleosynthetic components but rather reflect the different timescales and conditions of core formation (Touboul et al., 2007). 
There are three possible explanations for the $\mathrm{W}$ isotopic similarities of the Earth and Moon. First, the impactor just happened to have identical isotopic signatures to the Earth. Second, the bulk of the Moon was derived largely from the proto-Earth, implying that there is a fundamental error in current models of lunar origin. Third, the Moon isotopically re-equilibrated with the Earth following the giant impact, as has recently been proposed to account for the $\mathrm{O}$ isotope similarity of the Earth and Moon (Pahlevan and Stevenson, 2007).

Below we investigate the first of these possibilities for tungsten. Qualitatively, our argument is as follows. Lunar- to Mars-mass embryos generally form within a few Ma (Kokubo and Ida, 2000), while their growth into Earth-mass planets takes 10-100 Ma. Other things being equal, the Mars-mass moon-forming impactor should therefore develop more radiogenic mantle tungsten than the target (Earth). Since the present-day Moon actually has a higher $\mathrm{Hf} / \mathrm{W}$ ratio than the Earth, it should have even more radiogenic mantle tungsten. Thus, the fact that the Moon and Earth have identical mantle tungsten isotopic signatures is a potential argument for postimpact re-equilibration. To quantify this argument, we resort to dynamical N-body simulations in which tungsten isotopic evolution is tracked. We find that impactors having Moon-like Hf/W ratios always acquire more radiogenic mantle tungsten than the present-day Moon. Having examined various mixing scenarios to reduce this mismatch, we conclude that post-impact reequilibration is required to explain the present-day observations (unless the N-body accretion timescale or the lunar impact simulations are incorrect).

One advantage of this approach is that we can use physical (mass, semimajor axis) as well as isotopic observations to help reduce the non-unique nature of the Hf-W constraint. We also require our models to simultaneously produce both Earth-like and Mars-like bodies, since these are the only two major bodies for which we have isotopic measurements. To test the robustness of our results, we adopt several different models for how the Hf/W ratio varies between bodies (Section 4.1). We find that the results are very similar, because the logic outlined in the preceding paragraph does not depend on the model details. We also investigate (Section 3.3) the extent to which addition of proto-Earth (target) material to the impactor could result in more Moon-like results. 


\section{Methods}

\subsection{N-body accretion model}

The tungsten isotope calculations, described below, are carried out as a post-processing step on the results from a series of N-body simulations performed by O'Brien et al. ((O'Brien et al., 2006); hereafter OML06). These simulations are initiated with 25 Mars-mass $\left(0.093 M_{E}\right.$, where $M_{E}$ is the present-day mass of the Earth) planetary embryos and 1000 planetesimals 1/40th as massive spanning $0.3 \mathrm{AU}$ to $4 \mathrm{AU}$ in distance. Half the initial mass is contained in the embryos and half in the planetesimals. The orbits of all these bodies are tracked, with the small planetesimals perturbing the orbits of the embryos but not each other. When collisions occur they result in perfect mergers with angular momentum conserved. The orbits were tracked for $250 \mathrm{Ma}$. Surviving massive bodies are typically comprised of a few to $\sim 10$ embryos and several tens of planetesimals.

Two different suites of simulations were run. In the first suite (EJS1-4) Jupiter and Saturn are included on their current orbits. In the second suite (CJS1-4), Jupiter and Saturn are closer together and on zero-eccentricity orbits, to reproduce their hypothesized configuration prior to the Late Heavy Bombardment (Gomes et al., 2005). For both configurations, roughly half of the initial mass ends up in planets, with the remainder either ejected from the system or hitting the Sun. Both sets of simulations result in 2-4 roughly Earth-mass planets closer than 2.5 AU. The main differences between the two suites are that the EJS runs result in earlier termination of accretion, planets with lower eccentricities, a smaller planet near the position of Mars, and less mixing of material from the outer asteroid belt into the terrestrial planet zone.

Our N-body code neglects the effect of fragmentation. Impact erosion can lead to changes in bulk planetary chemistry (O'Neill and Palme, 2008), while fragmentation causes both the timescale of accretion and the final planet masses to change (Chambers, 2008). Although not included here, the chemical consequences of these processes are in principle easy to investigate.

\subsection{The ${ }^{182} \mathrm{Hf}-{ }^{182} \mathrm{~W}$ system: definitions and observational constraints}

Hafnium is lithophile and $\mathrm{W}$ is siderophile, resulting in strong fractionation during core formation, while ${ }^{182} \mathrm{Hf}$ is unstable and decays to ${ }^{182} \mathrm{~W}$ with a half-life of 9 Ma. As a result, early core formation results in mantles with excess ${ }^{182} \mathrm{~W}$ (i.e. a positive tungsten anomaly), with the magnitude of the 
excess depending on the timing of the event and the degree of fractionation (Kleine et al., 2009, e.g.). Tungsten isotopic evolution depends not only on when core formation happened but also on how efficiently the impactor material re-equilibrates with the target's mantle during each individual impact, and on how strongly tungsten is subsequently partitioned into the target's core (Jacobsen, 2005; Halliday, 2004; Kleine et al., 2004b; Nimmo and Agnor, 2006).

There are two main observational constraints provided by Hf-W data. The first is the ratio of Hf to $\mathrm{W}$ in the planetary mantle. Here we will define this ratio relative to a chondritic reference as follows:

$$
f^{H f / W}=\frac{\left(C^{180 H f} / C^{183 W}\right)}{\left(C^{180 H f} / C^{183 W}\right)_{C H U R}}-1
$$

where $C^{180 H f}$ and $C^{183 W}$ are the mantle concentrations of the two isotopes, and CHUR refers to an undifferentiated (chondritic) ratio.

The second is the mantle tungsten anomaly $\varepsilon_{W}$, defined here as

$$
\varepsilon_{W}=\left[\frac{\left(C^{182 W} / C^{183 W}\right)}{\left(C^{182 W} / C^{183 W}\right)_{C H U R}}-1\right] \times 10^{4}
$$

In the remainder of this work, "tungsten anomaly" will be assumed to refer to the mantle of the body in question. A chondritic body has $f^{H f / W}=\varepsilon_{W}=0$ by definition.

We take the chondritic ${ }^{180} \mathrm{Hf} /{ }^{183} \mathrm{~W}$ ratio to be 2.627 (equivalent to an Hf/W ratio of 1.04) (Kleine et al., 2009, 2004a). The bulk silicate Earth $f^{H f / W}$ value is based on the Th/W ratio of Earth's mantle (Newsom et al., 1996) and is $13.6 \pm 4.3$ (Kleine et al., 2009). For the bulk silicate Moon, the corresponding $f^{H f / W}$ value is based on the $\mathrm{U} / \mathrm{W}$ ratio of the lunar mantle (Palme and Rammensee, 1982) and is $21.4 \pm 1.7$. The FeO content of the lunar mantle is probably $\approx 13 \mathrm{wt} \%$ (Jones and Palme, 2000), while the lunar core size is uncertain, but is unlikely to exceed $2 \%$ of the lunar mass (Wieczorek et al., 2006). As discussed by (Nimmo and Kleine, 2007), the Hf/W for bulk silicate Mars is uncertain. Assuming $\mathrm{Hf}$ and Th are present in carbonaceous chondrite relative abundances, $f^{H f / W}=2.4 \pm 0.9$ (note that (Nimmo and Kleine, 2007) use a different definition of $f^{H f / W}$ to that employed here).

The present day tungsten anomaly for the Earth is $\varepsilon_{W}=1.9 \pm 0.1$ (Yin et al., 2002; Kleine et al., 2002; Schoenberg et al., 2002; Kleine et al., 2004a). Interpretation of the lunar $\varepsilon_{W}$ has been bedevilled by cosmogenic effects 
(e.g. Leya et al., 2000), but it has been shown that lunar metals preserve the indigenous $\mathrm{W}$ isotopic composition of their host rocks (Touboul et al., 2007; Kleine et al., 2005b). The lunar metal data reveal that the lunar mantle has a tungsten anomaly of $\varepsilon_{W}=2.0 \pm 0.1$, which is identical within error to the terrestrial value (Touboul et al., 2007, 2009). For Mars, the shergottites yield a tungsten anomaly of $\varepsilon_{W}=2.3 \pm 0.2$ (Kleine et al., 2004a; Foley et al., 2005). Nakhlites have larger ${ }^{182} \mathrm{~W}$ excesses but these most likely stem from an early differentiation within the martian mantle and hence do not only result from core formation (Kleine et al., 2004a; Foley et al., 2005). Hence, we take the tungsten anomaly of shergottites to represent that of the bulk martian mantle.

\subsection{Isotopic calculations}

Isotopic evolution is calculated using an approach based on Nimmo and Agnor ((Nimmo and Agnor, 2006); hereafter NA06). The evolution of four isotopes $\left({ }^{182} \mathrm{Hf},{ }^{182} \mathrm{~W},{ }^{180} \mathrm{Hf}\right.$ and ${ }^{183} \mathrm{~W}$, where the latter two are stable) is tracked for both the core and mantle of each body in the N-body simulation. Decay of the radioactive isotopes occurs continuously; when two bodies collide, the isotopic concentrations of the resulting body are calculated according to the scheme outlined below, which includes various mixing scenarios between the target's mantle and the impactor's core and mantle. All bodies are assumed to start undifferentiated. Bodies differentiate during the first impact, or at a preset time $t_{d i f f}$, whichever happens first. For Mars-sized or larger bodies early differentiation due to gravitational energy release is almost inevitable (Rubie et al., 2007); smaller bodies apparently underwent widespread early melting caused by ${ }^{26} \mathrm{Al}$ decay (Bizzarro et al., 2005; Kleine et al., 2005a).

We define the equilibration factor $k(0 \leq k \leq 1)$, which gives the fraction of the impactor core not added directly to the target core. Thus, $k=1$ indicates complete re-equilibration, and $k=0$ indicates no re-equilibration (core merging). We assume that the entire mantle undergoes equilibration with the metal, which may not be appropriate for small collisions in which impact heating is localized.

In addition to choosing a value for $k$, the isotopic calculations require a description of partitioning during core formation. Following differentiation, the elemental concentration in the silicate component $C_{m}$ is given by

$$
C_{m}=\frac{C}{D(1-y)+y}
$$


where $D$ is the metal-silicate partition coefficient, $C$ is the initial concentration, $y$ is the silicate mass fraction, and a similar equation holds for core concentrations.

The tungsten partition coefficient $D_{W}$ is not necessarily constant in either time or space, because the partitioning of $\mathrm{W}$ into the core depends on several factors including oxygen fugacity, temperature and pressure (Cottrell et al., 2009). Mars and the Earth exhibit very different Hf/W ratios, suggesting that spatial or temporal variations may be important. Because significant quantities of water are expected to be found only beyond a certain distance (the snow line) during accretion, the degree of oxidation is expected to increase with distance from the Sun (Pasek et al., 2005; Bond et al., 2009). Because reduced environments result in larger $D_{W}$ values (Cottrell et al., 2009), the $\mathrm{Hf} / \mathrm{W}$ ratio should decrease with increasing semi-major axis. Variability in oxidation state between different embryos is consistent with the variability of $\mathrm{Hf} / \mathrm{W}$ ratios seen in parent bodies of differentiated meteorites (Kleine et al., 2004a).

\subsection{Approach and assumptions}

We assume that $D_{W}$ for tungsten varies with radial position (see above). The mantle fractionation factor $f^{H f / W}$ is given by $f^{H f / W}=D_{W}(1-y) / y$, where $y$ is the silicate mass fraction (see (Jacobsen, 2005) eq. 49). The specific form of $D_{W}$ variation chosen (see Fig 1a) was designed to mimic the likely steep change in oxidation state at the snow line (Pasek et al., 2005), and to generate roughly Earth- and Mars-like values of $f^{H f / W}$ at the appropriate distances. In reality, of course, the location of the snow line probably varied with time and variations in oxidation state were likely more complicated (Kleine et al., 2009; Halliday, 2004; Wade and Wood, 2005); however, as discussed in Section 4.1 the exact functional form adopted does not significantly affect the final results.

For Hf, we take the $D$ value to be $10^{-4}$ (entirely lithophile) throughout. The initial isotopic concentrations assumed are identical to those used by NA06, but do not differ significantly from more recent estimates (Burkhardt et al., 2008). Except where stated otherwise, we assume that $t_{\text {diff }}=3 \mathrm{Ma}$; we investigate the sensitivity of the results to this assumption in Section 4.1.

We initially assume that the Moon forms entirely from impactor material. However, because numerical studies show that up to $40 \%$ of the Moon may be derived from the target (Canup, 2008), in Section 3.3 we investigate the effect of adding target material to the proto-Moon. 
We assume that all bodies have the same initial silicate:metal ratio $(y=0.68)$, appropriate for the terrestrial planets. In reality, this value is likely to vary somewhat between bodies, but NA06 showed that the likely variability does not significantly affect the final results.

A more serious shortcoming is that we assume that all impacts re-equilibrate to the same degree, that is, $k$ is fixed. While there are theoretical arguments that $k$ is likely to vary depending on the mass and mass ratio of the impactor (Rubie et al., 2003), how re-equilibration proceeds is currently poorly understood.

\section{Results}

\subsection{General results}

Figure 1 and Table 1 summarize the physical and isotopic results from all eight N-body simulations, for bodies with masses $\geq 0.2 M_{E}$ and $\geq 0.7 M_{E}$, respectively. These calculations assumed $k=0.5$.

Fig 1a plots the variation in mantle $f^{H f / W}$ against final semi-major axis for surviving bodies. The solid line depicts the initial assumed variation, and the solid bars show the measured values for Mars and the Earth. Although there is some scatter, the initial variation from high to low $f^{H f / W}$ with increasing semi-major axis is retained, indicating that most bodies grow mainly via collisions with nearby neighbours (Wetherill, 1994). Bodies resembling both Mars and the Earth are generated.

Fig $1 \mathrm{~b}$ shows how the final mantle tungsten anomaly varies with planetary mass. Earth-like and Mars-like $\varepsilon_{W}$ values are generated; all bodies plotted have masses larger than that of Mars, because the starting mass of individual embryos is approximately one Mars-mass. On average, the tungsten anomaly decreases slightly with increasing mass. This is because larger bodies have suffered more collisions, and under the assumption of partial re-equilibration each collision drives $\varepsilon_{W}$ towards zero.

Fig $1 \mathrm{c}$ shows how tungsten anomaly varies with mantle $f^{H f / W}$. Both Earth- and Mars-like values are reproduced. Surprisingly, however, the expected positive correlation is not seen. This effect is partly due to the stochastic nature of accretion. More important, however, is a trade-off between two effects. As seen in Fig 1d, the minimum value of $f^{H f / W}$ is larger for the larger bodies, because more material is available for growth in the inner part of the model domain (where $f^{H f / W}$ is highest, by assumption). Thus, larger bodies 
should develop larger tungsten anomalies. On the other hand, larger bodies suffer more collisions, which drive the tungsten anomaly down (Fig 1b). These two effects roughly cancel each other out, providing a potential explanation for why Mars and Earth have similar tungsten anomalies in spite of their different accretion history.

Three further dynamical aspects of the accretion models are enumerated in Table 1. First, for these giant impacts the impactor:target mass ratio $\gamma$ is usually smaller for larger bodies. This is partly because the largest bodies become gravitationally isolated (typically separated by several tens of Hill radii; (Agnor et al., 1999)) and are thus unlikely to encounter other bodies of comparable size. Additionally, larger bodies tend to grow faster than smaller bodies, so as they grow they encounter progressively smaller impactors. In any event, Table 1 shows that a model body with a final mass $\approx 1 M_{E}$ results in $\gamma \approx 0.1-0.2$, which is consistent with estimated impactor masses for the Moon-forming impact (Canup, 2008).

Second, most of the final large impacts occur in the first $50 \mathrm{Ma}$, but there is a weak trend: large bodies tend to experience the final big impact later than small bodies. This effect is most likely because larger bodies have a larger gravitational cross-section, making a late encounter with a stray embryo more likely. However, because of the stochastic nature of the accretion process, this trend shows considerable scatter. For bodies in the range 0.8-1.2 $M_{E}$, Table 1 demonstrates that the final large impact can occur as early as $14 \mathrm{Ma}$ or as late as $232 \mathrm{Ma}$. Thus, from a dynamical point of view the timing of the Moon-forming impact is poorly constrained.

Third, the fraction of mass added after the final giant impact $\left(\gamma_{v n r}\right)$ shows a strong inverse correlation with the timing of the final giant impact: an early final collision results in significant amounts of material being added later through collisions with planetesimals. The effect of this late addition of material is generally to reduce the final tungsten anomaly of the target body. This material should not to be confused with the "late veneer" which is commonly invoked to explain the overabundance of highly siderophile elements in the Earth's mantle, and which is assumed not to involve core-mantle partitioning. As discussed below (Section 4.2), based on geochemical grounds the permissible value of $\gamma_{v n r}$ is $<5 \%$, such that the value of $\gamma_{v n r}$ produced in each of the simulations can be used to distinguish between more and less realistic cases.

For our assumed initial variation in $f^{H f / W}$ (Fig 1a), Earth- and Marslike bodies can be produced. However, the model results are sensitive to the 
re-equilibration factor $k$ chosen. Fig. 2 plots the mean value and standard deviation of $\varepsilon_{W}$ for Earth-like bodies (circles) as $k$ is varied. Here results from EJS and CJS simulations are not significantly different and are lumped together, but only bodies with masses $\geq 0.7 M_{E}$ are used. Circles with solid lines use the $f^{H f / W}$ variation shown in Fig 1a, and result in mean $f^{H f / W}$ values denoted by the crosses. Circles with dashed lines assume a constant Earth-like $f^{H f / W}$ of 13.8 . Taking the constant and variable $f^{H f / W}$ cases together, re-equilibration factors in the range $k=0.3-0.8$ result in Earth-like tungsten anomalies.

Fig. 2 shows the effect of varying $k$ on the tungsten anomaly, but assumes a fixed shape for the $f^{H f / W}$ curve (Fig 1a). Since there is a potential tradeoff between $k$ and $f^{H f / W}$ (Kleine et al., 2009), we also investigated the effects of scaling the $f^{H f / W}$ curve by a constant multiplicative factor $F$. For near-complete re-equilibration $(k=0.8)$ we found that even with $F=2$ we still obtained model tungsten anomalies more than one standard deviation smaller than the observed terrestrial value. For near-complete core merging $(k=0.2)$, reducing $F$ to $\leq 0.6$ resulted in an $f^{H f / W}$ value more than one standard deviation smaller than that observed, without reducing the model tungsten anomaly to the observed value. We therefore conclude that our estimated range $0.3 \leq k \leq 0.8$ is robust to likely variations in the shape of the $f^{H f / W}$ curve. Neither complete re-equilibration nor complete core merging can generate Earth-like results in these models; as a result, the calculations below generally assume $k=0.5$.

\subsection{Moon-forming impacts}

Having demonstrated that our nominal model can generate Earth- and Mars-like bodies, we now turn to the issue of the Moon-forming impact. Figure 3a plots $\varepsilon_{W}$ for the last giant impactor, compared with $\varepsilon_{W}$ for the final (post-impact) body, for both the constant and variable $f^{H f / W}$ cases. Here all the anomalies plotted are the present-day value of $\varepsilon_{W}$. The grey shaded box is the measured Earth/Moon tungsten anomaly. The standard theory of Moon formation (Canup, 2008) dictates that the tungsten anomalies of the impactor and final body should approximate those measured for the Moon and Earth, respectively; in Section 3.3 below we explore the effects of modifications to this theory.

Despite the scatter, the model results show a general trend with larger impactor tungsten anomalies resulting in larger tungsten anomalies for the final body. This correlation is not surprising, since incomplete re-equilibration 
means that the pre-existing anomaly in the impactor contributes to that of the target. For the constant $f^{H f / W}$ case (triangles), all of the impactors have tungsten anomalies $\left(\varepsilon_{W} \geq 5\right)$ larger than the Earth/Moon value $\left(\varepsilon_{W} \approx 2\right)$. For the variable $f^{H f / W}$ case (circles), some impactor/target pairs have $\varepsilon_{W}$ values similar to those of the Earth/Moon system. However, Fig 3b plots the impactor $f^{H f / W}$ against the impactor tungsten anomaly and demonstrates that those impactors with low enough tungsten anomalies have $f^{H f / W}$ values much smaller than that of the lunar mantle.

For the variable $f^{H f / W}$ case, Fig 3 shows that the EJS runs have systematically higher Hf/W ratios and tungsten anomalies than CJS runs. This is partly because EJS runs result in the incorporation of less material (with low $\mathrm{Hf} / \mathrm{W}$ ) from the outer edge of the model domain, and partly because the final collision usually happens earlier in these runs (Table 1).

Neither the constant nor the variable $f^{H f / W}$ cases can reproduce the Earth/Moon observations, for the following reason. Because they are small, most impactors complete their growth early - the median time for an impactor to reach $90 \%$ of its final mass $\left(T_{90 \%}\right)$ is $<10$ Ma (Table 1$)$. Early growth and a relatively high $\mathrm{Hf} / \mathrm{W}$ ratio results in a large tungsten amomaly. For instance, an impactor which differentiates after $10 \mathrm{Ma}$ and has $f^{H f / W}=20$, appropriate to the Moon, will generate a tungsten anomaly of about 14 (Jacobsen, 2005, eq. 13). The lunar combination of relatively high $f^{H f / W}$ and relatively low $\varepsilon_{W}$ is impossible to reproduce for bodies which finished accreting within the first $\sim 10$ Ma.

\subsection{Constraints on lunar origin: evidence for Earth-Moon equilibration?}

For reasons explained above, our models are incapable of generating the relatively low $\varepsilon_{W}$ of the Moon while simultaneously satisfying its high $\mathrm{Hf} / \mathrm{W}$ ratio. It is therefore important to investigate whether a low $f^{H f / W}$ value of an impactor mantle (and, hence, the bulk Moon) could have been modified to the high $f^{H f / W}$ value observed in the lunar mantle by formation of a lunar core.

Evaluating such a model requires knowledge of $D_{W}$ appropriate for the lunar mantle. Based on the FeO content of lunar basalts, the oxygen fugacity of the Moon has been estimated to be approximately one log unit below the iron-wustite buffer $(\Delta \mathrm{IW} \approx-1)$ or even somewhat higher (Jones and Palme, 2000). $D$ for tungsten at an oxygen fugacity of $\Delta \mathrm{IW}=-1$ is $\approx 30$ (Cottrell et al., 2009; Walter et al., 2000), and decreases with increasing oxygen fugacity. This value for $D_{W}$ can be compared to that required to 
generate the high $f^{H f / W}$ of the lunar mantle by differentiation of a model Moon with a lower bulk $f^{H f / W}$.

Impact simulations show that the bulk Moon should consist mainly of impactor mantle (Canup, 2008), but there are two other sources that might have contributed material: (i) the impactor core, a small fraction of which may have been incorporated into the Moon, and (ii) the proto-Earth's mantle. The fraction of proto-Earth mantle material that ended up in the Moon is not well constrained. However, to achieve the correct lunar mass, current models suggest that at most $40 \%$ of the Moon was derived from the Earth (e.g. Fig. 5 of (Canup, 2008)).

Whether the Moon contains any material from the impactor core is unclear. Depending on the composition of the impactor mantle, some impactor core material might be required to deliver the Fe that now resides in the small lunar core. However, using the $\mathrm{Fe}-\mathrm{FeO}$ partition coefficient of $\approx 6$ at an oxygen fugacity of $\Delta \mathrm{IW}=-1$ (Holzheid and Palme, 1996) and a $2 \%$ lunar core results in an iron content of the bulk Moon of $\approx 11$ wt.- $\%$ Fe, well below the Fe content of a Mars-like impactor mantle with $\approx 14$ wt.- $\%$ Fe (Taylor et al., 2006). Thus, a Mars-like impactor mantle contains sufficient Fe to be consistent with the lunar mantle $\mathrm{FeO}$ content and the presence of a small lunar core. Given the uncertainties, and to investigate the effect of variations in the composition of the bulk Moon, we used two different compositions for the bulk Moon in our calculations: $98 \%$ impactor mantle $+2 \%$ impactor core; and $49 \%$ impactor mantle $+1 \%$ impactor core $+50 \%$ proto-Earth (target) mantle.

Figs. 4a-b show the tungsten anomaly of the model lunar mantle after differentiation as a function of its $f^{H f / W}$ calculated for two different values of $D_{W}$ and our two different assumed bulk lunar compositions. In neither case can the nominal $D_{W}=30$ value simultaneously satisfy the observed lunar tungsten anomaly and $f^{H f / W}$. For the pure impactor case (Fig 4a), even a $D_{W}$ of 150 is insufficient to satisfy both constraints at once. Addition of target mantle, which tends to have a lower tungsten anomaly, helps matters somewhat (Fig 4b). However, even in this case a $D_{W}$ of 60 , double that of the expected value, is required to permit even a single model result (labelled A in Fig 4b) to resemble the observed lunar parameters. Furthermore, this particular case results in a final target (Earth) tungsten anomaly which is too small (Table 1, Fig 3).

It has been argued (Newsom and Drake, 1982) that if lunar core formation took place under low degrees of silicate melting, W would have been efficiently 
separated into the lunar core. Lunar core formation could thus bring down the abundance of $\mathrm{W}$ in the bulk silicate Moon to the observed value even if the bulk Moon had chondritic Hf/W. However, the high initial temperatures of the proto-lunar disk suggest that lunar core formation took place in an almost fully molten Moon (Pritchard and Stevenson, 2000). Thus, unless mixing with proto-Earth material was important, the high $\mathrm{Hf} / \mathrm{W}$ ratio of the lunar mantle cannot simply be explained by lunar core formation but reflects the high $\mathrm{Hf} / \mathrm{W}$ of the proto-lunar material.

A related question is the robustness of the inferred $\mathrm{Hf} / \mathrm{W}$ ratio of the lunar mantle. This estimate is derived from the measured and constant $\mathrm{U} / \mathrm{W}$ ratio of a variety of lunar rocks and by assuming that the bulk Moon has constant ratios of refractory lithophile elements, in this case Hf and U. However, Fegley and Cameron (1986) observed that in highly oxidized environments (such as partially vapourized material resulting from planetary collisions), U may be preferentially lost relative to other refractory elements due to its increased volatility. If $\mathrm{U}$ were lost from the Moon, its $\mathrm{Hf} / \mathrm{U}$ ratio would be higher than chondritic. However, both the Th/U (Tera and Wasserburg, 1972) and the $\mathrm{La} / \mathrm{U}$ (Palme and Rammensee, 1982) ratios of the Moon are similar to those of chondrites, suggesting that preferential loss of $U$ did not in fact occur.

A final possibility is that the timescales produced by the N-body model are incorrect, perhaps due to the neglected effects of "hit and run" collisions (Asphaug et al., 2006) or fragmentation (Chambers, 2008). Increasing the accretion timescale would reduce $\varepsilon_{W}$ for a given $f^{H f / W}$, thus making Moonlike results more likely. We therefore carried out a set of calculations in which the accretion timescale is multiplied by a constant factor of 3 . These results are plotted in Fig $3 b$ (triangles), showing that an artificially increased timescale does improve the fit between model and observations, although it does not solve the problem entirely.

With this timescale and the 49\%/1\%/50\% mixing scenario, one model did produce a single outcome (EJS4.4) with an Earth-like target and a Moonlike impactor for $D_{W}=30$ and $k=0.3$. The target and impactor values were $f^{H f / W}=20.3, \varepsilon_{W}=1.8$ and $f^{H f / W}=17.8, \varepsilon_{W}=2.0$, respectively. Thus, if the N-body timescales are wrong by a factor of three, it may be possible to avoid the requirement for Earth-Moon re-equilibration.

We conclude that with the nominal N-body timescale, the observed $f^{H f} / W$ and $\varepsilon_{W}$ values of the Earth and Moon cannot be obtained in a self-consistent fashion from our model. This result suggests that the Moon isotopically equilibrated with the Earth's mantle in the aftermath of the giant impact (cf. 
Pahlevan and Stevenson, 2007). Alternatively, there could be an error either in the current models which show the Moon being derived primarily from the impactor mantle, or in the accretion timescales obtained from the N-body simulations. Distinguishing between these two possibilities will require both (i) evaluating whether Earth-Moon equilibration is possible for a refractory element such as $\mathrm{W}$ and (ii) further improvements in simulations of accretion and the giant impact.

\section{Discussion}

We may summarize the results of the preceding section as follows. First, a re-equilibration factor of $0.3 \leq k \leq 0.8$ is required to generate Earth-like Hf/W ratios and tungsten isotope anomalies (Fig. 2). This holds true in both the variable $D_{W}$ and constant $D_{W}$ cases. Second, using the nominal N-body

timescale, none of our models produce Moon-like values of both $f^{H f / W}$ and $\varepsilon_{W}$ within a single impactor (Figs 3,4$)$.

\subsection{Effects of varying parameters}

Several parameters might affect our results. Our nominal model assumes $k=0.5$. We generated plots similar to Figs 3 and $4 \mathrm{~b}$ using $k=0.3$ and $k=0.8$, but in no case did we find a model Moon which matched the observed $\varepsilon_{W}$ and $f^{H f / W}$ values for a lunar $D_{W}$ of 30 .

Reducing the differentiation time $t_{\text {diff }}$ from $3 \mathrm{Ma}$ to $0 \mathrm{Ma}$ results in a roughly $10 \%$ increase in the final body tungsten anomalies, too small to be significant. Increasing $t_{\text {diff }}$ means bodies will not differentiate until their first collision. An impactor with $f^{H f / W}=20$ undergoing its first collision at $35 \mathrm{Ma}$ would generate a Moon-like tungsten anomaly, but as Table 1 shows, all but one impactors finish their accretion well before this time.

Our nominal model assumed a spatially-variable $D_{W}$ resulting in the $f^{H f / W}$ variation shown in Fig 1a. Although qualitatively reasonable, the quantitative form is ad hoc. We therefore also used two alternative models to investigate the sensitivity of our results to the starting assumptions.

The first model simply assumes a constant, Earth-like $D_{W}$. This approach has the disadvantage that Mars-like bodies cannot be produced, but has the virtue of simplicity. As shown in Fig 3, our main conclusion - that Moon-like $\varepsilon_{W}$ and $f^{H f / W}$ values cannot be obtained simultaneously - is true for both the spatially-varying and the constant $D_{W}$ assumption. 
Our second model (not shown) is more complicated, and assumes that $D_{W}$ increases in a linear or power-law fashion with increasing mass. This is motivated by the need to move from Mars-like to Earth-like Hf/W ratios as mass increases. Such an increase is not supported by the proposed increase in oxidation of the Earth through time (Wade and Wood, 2005; Wood et al., 2008) but serves to demonstrate the insensitivity of our results to the specific model adopted. For models in which Earth-like and Mars-mass bodies attain Earth- and Mars-like $f^{H f / W}$ values, we find that $0.2 \leq k \leq 0.7$ and that (as with our nominal and first alternative models) none of these latter models simultaneously generate Moon-like $\varepsilon_{W}$ and $f^{H f / W}$ values, even after taking mixing of the kind shown in Fig 4 into account.

One interesting consequence of our nominal model is that later collisions with Earth-like bodies involve impactors that are more oxidized (lower

$f^{H f / W}$ ) than earlier impactors. This is simply a consequence of the feedingzone of the target expanding with time, and thus sampling bodies at progressively greater radial distances. Hence, our nominal model has at least the potential to explain the inferred temporal increase in terrestrial oxidation state (Wade and Wood, 2005; Wood et al., 2008).

\subsection{Comparison of EJS and CJS runs}

Table 1 shows that Earth-like target tungsten anomalies can be generated for a wide range of final giant impact times, especially if $k$ is allowed to vary. Thus, our results strengthen earlier arguments that the tungsten anomaly of Earth alone provides only weak constraints on the timing of Earth's core formation and formation of the Moon (Kleine et al., 2009; Halliday, 2004; Kleine et al., 2004a).

However, the CJS runs generally result in later final giant impacts, and thus more Earth/Moon-like tungsten anomalies (Fig 3). A similar comment also applies when mixing of the target and impactor are taken into account (Fig 4). It therefore appears that the CJS runs provide a better match to the observations.

Furthermore, in the EJS simulations, 3-19 \% of Earth's mass is added after the final Moon-forming impact, whereas in most of the CJS simulations the fraction of material added after the final giant impact $\left(\gamma_{v n r}\right.$ in Table 1$)$ is much smaller. Canup and Asphaug (Canup and Asphaug, 2001) argued that the Fe-poor composition of the Moon and the small size of its core requires that only a small fraction of Earth's mass could have been added after the giant impact. Using an Earth/Moon mass influx ratio of $\approx 30$ (Stewart, 
2000) and assuming that the impactor has an Fe content similar to that of the Earth ( $\approx 31$ wt. \%), Earth could not have accreted more than $\approx 3$ $\%$ of its mass before the Moon would have gained more than 0.02 lunar masses of $\mathrm{Fe}$, equivalent to the upper limit on lunar core size. Based on Table 1, this constraint on $\gamma_{v n r}$ rules out final giant impacts earlier than $\approx 60 \mathrm{Ma}$, consistent with other estimates of the Moon's age (e.g. Touboul et al., 2007, 2009; Carlson and Lugmair, 1988). These geochemical arguments for the addition of only a small fraction of Earth's mass after the Moonforming impact are consistent with results of most of the CJS simulations but are inconsistent with all but one of the EJS simulations. This particular argument assumes that collisions with the Moon result in mergers, which may not be correct owing to the Moon's lower escape velocity. Nonetheless, the CJS simulations seem to be more capable of reproducing the observed geochemical and Hf-W characteristics of the Earth and Moon.

\subsection{Incomplete metal-silicate equilibration during large impacts}

Our results indicate that partial re-equilibration is required, a conclusion that has also been drawn in earlier studies (Kleine et al., 2004b,a). NA06 concluded that Earth-like values could be produced even with complete reequilibration $(k=1)$. By contrast, we find that with either a variable or a constant (Earth-like) value of $D_{W}$, complete re-equilibration is ruled out (Fig 2). The most likely reason for this discrepancy is that NA06 used Nbody simulations with 22 embryos and no planetesimals. In the present simulations, surviving planets experience a steady stream of planetesimal collisions, each of which reduces the tungsten anomaly a little. Since the planetesimals make up half the initial mass in the system, this reduction is sufficiently pronounced that it requires a corresponding reduction in the efficiency of re-equilibration $(k<1)$ to yield Earth-like tungsten anomalies.

The physical significance of incomplete equilibration is not yet clear. As discussed in (Rubie et al., 2007, 2003), complete equilibration likely requires the emulsification of $\sim 1000 \mathrm{~km}$-scale impactor cores down to cm-scale droplets during their transit through the target mantle. Whether this is physically likely is difficult to model numerically, due to the enormous range in lengthscales, but might be investigated with laboratory experiments (Olson and Weeraratne, 2008).

Incomplete metal-silicate equilibration during large impacts has important implications for understanding the distribution of siderophile elements in Earth's mantle. Several authors have found that the observed siderophile 
element abundances are consistent with those predicted from metal-silicate partition coefficients, if metal-silicate equilibrium was achieved in a deep magma ocean (e.g. Wood et al., 2008; Righter, 2003). These results are not consistent with the evidence for incomplete metal-silicate equilibration presented in this study. Whether mantle siderophile element abundances can be satisfied with an incomplete equilibration model of the kind proposed here is evidently an important question to address in future work.

\section{Conclusions}

This study demonstrates that N-body simulations can generate bodies that have both physical and chemical characteristics (mass, semi-major axis, $\left.f^{H f / W}, \varepsilon_{W}\right)$ similar to Earth or Mars. Generating Earth-like characteristics requires partial re-equilibration $(0.3 \leq k \leq 0.8)$, irrespective of the specific $D_{W}$ variation adopted.

The CJS simulations produce results that are more compatible with the tungsten characteristics of the Earth and Moon than the EJS simulations. This result is consistent with the so-called Nice model of early solar system development (Gomes et al., 2005), which predicts that Jupiter and Saturn were initially on circular orbits.

None of our models succeeded in generating impactors with both Moonlike $f^{H f / W}$ and Moon-like $\varepsilon_{W}$ for the nominal accretion timescale. The indistinguishable $\varepsilon_{W}$ values of the lunar and terrestrial mantles could arise if the Moon predominantly consists of material that was ejected from the protoEarth during the giant impact, or if current N-body accretion timescales are too rapid. Alternatively, the $\mathrm{W}$ isotopes could have equilibrated between the lunar magma disk and Earth's mantle. If current numerical models for the Moon-forming impact and N-body accretion are realistic, then post-impact Earth-Moon equilibration is required. It will thus be important to investigate the circumstances under which equilibration of a refractory element such as $\mathrm{W}$ is possible. Another question of considerable interest is the extent to which isotope similarities beween the Earth and Moon of other refractory elements (e.g. Ti, Cr) can also be explained by post-impact equilibration.

Acknowledgements We thank Rick Carlson, John Chambers, Kaveh Pahlevan and three anonymous reviewers for helpful comments. This study was supported in part by NASA PG\&G and the SNF. 


\section{References}

Agnor, C.B., Canup, R.M., Levison, H.F., 1999. On the character and consequences of large impacts in the late stage of terrestrial planet formation. Icarus 142, 219-237.

Asphaug, E., Agnor, C.B., Williams, Q., 2006. Hit-and-run planetary collisions. Nature 439, 155-160.

Bizzarro, M., Baker, J.A., Haack, H., Lundgaard, K.L., 2005. Rapid timescales for accretion and melting of differentiated planetesimals inferred from Al26-Mg26 chronometry. Astrophys. J. 632, L41-44.

Bond, J.C., Lauretta, D.S., O'Brien, D.P., 2009. Making the Earth: Combining dynamics and chemistry in the Solar System. Icarus, in press.

Burkhardt, C., Kleine, T., Bourdon, B., Palme, H., Zipfel, J., Friedrich, J.M., Ebel, D.S., 2008. Hf-W mineral isochron for Ca,Al-rich inclusions: Age of the solar system and the timing of core formation in planetesimals. Geochim. Cosmochim. Acta 72, 6177-6197.

Canup, R.M., 2008. Accretion of the Earth. Phil. Trans. R. Soc. Lond. A $366,4061-4075$.

Canup, R.M., Asphaug, E., 2001. Origin of the Moon in a giant impact near the end of the Earth's formation. Nature 412, 708-712.

Carlson, R.W., Lugmair, G.W., 1988. The age of ferroan anorthosite 60025 - oldest crust on a young Moon. Earth Planet. Sci. Lett. 90, 119-130.

Chambers, J., 2008. Oligarchic growth with migration and fragmentation. Icarus 198, 256-273.

Cottrell, E., Walter, M.J., Walker, D., 2009. Metal-silicate paritioning of tungsten at high pressure and temperature: Implications for equilibrium core formation in Earth. Earth Planet. Sci. Lett. 281, 275-287.

Fegley, B., Cameron, A.G.W., 1986. A vapourization model for iron/silicate fractionation in the Mercury protoplanet. Earth Planet. Sci. Lett. 82, $207-222$. 
Foley, C., Wadhwa, M., Borg, L.E., Janney, P.E., Hines, R., Grove, T.L., 2005. The early differentiation of Mars from 182-W-142-Nd isotope systematics in the SNC meteorites. Geochim. Cosmochim. Acta 69, 45574571 .

Gomes, R., Levison, H.F., Tsiganis, K., Morbidelli, A., 2005. Origin of the cataclysmic Late Heavy Bombardment period of the terrestrial planets. Nature 435, 466-469.

Halliday, A., 2004. Mixing, volatile loss and compositional change during impact-driven accretion of the Earth. Nature 427, 505-509.

Holzheid, A., Palme, H., 1996. The influence of FeO on the solubilities of cobalt and nickel in silicate melts. Geochim. Cosmochim. Acta 60, 11811193.

Jacobsen, S.B., 2005. The Hf-W isotopic system and the origin of the Earth and Moon. Ann. Rev. Earth Planet. Sci. 33, 531-570.

Jones, J.E., Palme, H., 2000. Geochemical constraints on the origin of the Earth and Moon, in: Canup, R.M., Righter, K. (Eds.), Origin of the Earth and Moon, Univ. of Arizona Press. pp. 197-216.

Kleine, T., Mezger, K., Munker, C., Palme, H., Bischoff, A., 2004a. ${ }^{182} \mathrm{Hf}-{ }^{182} \mathrm{~W}$ isotope systematics of chondrites, eucrites and martian meteorites: Chronology of core formation and early mantle differentiation in Vesta and Mars. Geochim. Cosmochim. Acta 68, 2935-2946.

Kleine, T., Mezger, K., Palme, H., Munker, C., 2004b. The W isotope evolution of the bulk silicate Earth: constraints on the timing and mechanisms of core formation and accretion. Earth Planet. Sci. Lett. 228, 109-123.

Kleine, T., Mezger, K., Palme, H., Scherer, E., Munker, C., 2005a. Early core formation in asteroids and late accretion of chondrite parent bodies: Evidence from ${ }^{182} \mathrm{Hf}-{ }^{182} \mathrm{~W}$ in CAIs, metal-rich chondrites, and iron meteorites. Geochim. Cosmochim. Acta 69, 5805-5818.

Kleine, T., Munker, C., Mezger, K., Palme, H., 2002. Rapid accretion and early core formation on asteroids and the terrestrial planets from $\mathrm{Hf}-\mathrm{W}$ chronometry. Nature 418, 952-955. 
Kleine, T., Palme, H., Mezger, K., Halliday, A.N., 2005b. Hf-W chronometry of lunar metals and the age and early differentiation of the Moon. Science 310, 1671-1674.

Kleine, T., Touboul, M., Bourdon, B., Nimmo, F., Mezger, K., Palme, H., Jacobsen, S.B., Yin, Q.Z., Halliday, A.N., 2009. Hf-W chronology of the accretion and early evolution of asteroids and terrestrial planets. Geochim. Cosmochim. Acta 73, 5150-5188.

Kokubo, E., Ida, S., 2000. Formation of protoplanets from planetesimals in the solar nebula. Icarus 143, 15-27.

Leya, I., Schonbachler, M., Wiechert, U., Krahenbuhl, U., Halliday, A.N., 2008. Titanium isotopes and the radial heterogeneity of the solar system. Earth Planet. Sci. Lett. 266, 233-244.

Leya, I., Wieler, R., Halliday, A.N., 2000. Cosmic-ray production of tungsten isotopes in lunar samples and meteorites and its implications for hf-w cosmochemistry. Earth Planet. Sci. Lett. 175, 1-12.

Lugmair, G.W., Shukolyukov, A., 1998. Early solar system timescales according to Mn-53-Cr-53 systematics. Geochim. Cosmochim. Acta 62, 28632886.

Newsom, H.E., Drake, M.J., 1982. Constraints on the Moon's origin from the partitioning behaviour of tungsten. Nature 297, 210-212.

Newsom, H.E., Sims, K.W.W., Noll, P.D., Jaeger, W.L., Maehr, S.A., Beserra, T.B., 1996. The depletion of tungsten in the bulk silicate Earth: constraints on core formation. Geochim. Cosmochim. Acta 60, 1155-1169.

Nimmo, F., Agnor, C.B., 2006. Isotopic outcomes of N-body accretion simulations: Constraints on equilibration processes during large impacts from Hf/W observations. Earth Planet. Sci. Lett. 243, 26-43.

Nimmo, F., Kleine, T., 2007. How rapidly did Mars accrete? uncertainties in the Hf-W timing of core formation. Icarus 191, 497-504.

O'Brien, D.P., Morbidelli, A., Levison, H.F., 2006. Terrestrial planet formation with strong dynamical friction. Icarus 184, 39-58. 
Olson, P., Weeraratne, D., 2008. Experiments on metal-silicate plumes and core formation. Phil. Trans. R. Soc. Lond. A 366, 4253-4272.

O'Neill, H.S.C., Palme, H., 2008. Collisional erosion and the non-chondritic composition of the terrestrial planets. Phil. Trans. R. Soc. Lond. A 366, 4205-4238.

Pahlevan, K., Stevenson, D.J., 2007. Possible origin of the geochemical similarity of the Earth and Moon. Earth Planet. Sci. Lett. 262, 438-449.

Palme, H., Rammensee, W., 1982. The significance of $\mathrm{W}$ in planetary differentiation processes: Evidence from new data on eucrites. Proc. 12th Lunar Planet. Sci. Conf. , 949-964.

Pasek, M.A., Milsom, J.A., Ciesla, F.J., Lauretta, D.S., Sharp, C.M., Lunine, J.I., 2005. Sulfur chemistry with time-varying oxygen abundance during Solar System formation. Icarus 175, 1-14.

Pritchard, M.E., Stevenson, D.J., 2000. Thermal aspects of a lunar origin by giant impact, in: Canup, R.M., Righter, K. (Eds.), Origin of the Earth and Moon, Univ. of Arizona Press. pp. 179-196.

Righter, K., 2003. Metal-silicate partitioning of siderophile elements and core formation in the early Earth. Ann. Rev. Earth Planet. Sci. 31, 135-174.

Rubie, D.C., Melosh, H.J., Reid, J.E., Liebske, C., Righter, K., 2003. Mechanisms of metal-silicate equilibration in the terrestrial magma ocean. Earth Planet. Sci. Lett. 205, 239-255.

Rubie, D.C., Nimmo, F., Melosh, H.J., 2007. Formation of the Earth's core, in: Schubert, G. (Ed.), Treatise Geophysics v.9, Elsevier. pp. 51-90.

Schoenberg, R., Kamber, B.S., Collersen, K.D., Eugster, O., 2002. New $\mathrm{W}$-isotopic evidence for rapid terrestrial accretion and very early core formation. Geochim. Cosmochim. Acta 66, 3151-3160.

Stewart, G.R., 2000. Outstanding questions for the giant impact hypothesis, in: Canup, R.M., Righter, K. (Eds.), Origin of the Earth and Moon, Univ. of Arizona Press. pp. 217-226.

Taylor, G.J., et al., 2006. Bulk composition and early differentiation of Mars. J. Geophys. Res. 111, E03S10. 
Tera, F., Wasserburg, G.J., 1972. U-th-pb systematics in three Apollo 14 basalts and the problem of initial $\mathrm{Pb}$ in lunar rocks. Earth Planet. Sci. Lett. 14, 281-304.

Touboul, M., Kleine, T., Bourdon, B., Palme, H., Wieler, R., 2007. Late formation and prolonged differentiation of the Moon inferred from $\mathrm{W}$ isotopes in lunar metals. Nature 450, 1206-1209.

Touboul, M., Kleine, T., Bourdon, B., Palme, H., Wieler, R., 2009. Tungsten isotopes in ferroan anorthosites: Implications for the age of the Moon and lifetime of its magma ocean. Icarus 199, 245-249.

Trinquier, A., Birck, J.L., Allegre, C.J., Gopel, C., Ulfbeck, D., 2008. Mn53-Cr-53 systematics of the early solar system revisited. Geochim. Cosmochim. Acta 72, 5146-5163.

Wade, J., Wood, B.J., 2005. Core formation and the oxidation state of the Earth. Earth Planet. Sci. Lett. 236, 78-95.

Walter, M.J., Newsom, H.E., Ertel, W., Holzheid, A., 2000. Siderophile elements in the Earth and Moon: metal/silicate partitioning and implications for core formation, in: Canup, R.M., Righter, K. (Eds.), Origin of the Earth and Moon, Univ. of Arizona Press. pp. 265-290.

Wetherill, G.W., 1994. Provenance of the terrestrial planets. Geochim. Cosmochim. Acta 58, 4513-4520.

Wiechert, U., Halliday, A.N., Lee, D.C., Snyder, G.A., Taylor, L.A., Rumble, D., 2001. Oxygen isotopes and the Moon-forming giant impact. Science 294, 345-348.

Wieczorek, M.A., et al., 2006. The constitution and structure of the lunar interior. Rev. Mineralogy Geochem. 60, 221-364.

Wood, B., Wade, J., Kilburn, M.R., 2008. Core formation and the oxidation state of the Earth: Additional constraints from $\mathrm{Nb}, \mathrm{V}$ and $\mathrm{Cr}$ partitioning. Geochim. Cosmochim. Acta 72, 1415-1426.

Yin, Q.Z., Jacobsen, S.B., Yamashita, K., Blichert-Toft, J., Telouk, P., Albarede, F., 2002. A short timescale for terrestrial planet formation from Hf-W chronometry of meteorites. Nature 418, 949-952. 


\begin{tabular}{lrrrrrrrrr} 
Particle & $M_{f}$ & $\begin{array}{r}T_{i m p} \\
(\mathrm{Ma})\end{array}$ & $\gamma$ & $\begin{array}{r}\gamma_{v n r} \\
\%\end{array}$ & $\begin{array}{r}\varepsilon_{W} \\
\text { tar. }\end{array}$ & $\begin{array}{r}\varepsilon_{W} \\
\text { imp. }\end{array}$ & $\begin{array}{r}f f / W \\
\text { tar. }\end{array}$ & $\begin{array}{r}f^{H f / W} \\
\text { imp. }\end{array}$ & $\begin{array}{r}T_{90 \%} \\
(\mathrm{Ma})\end{array}$ \\
\hline CJS1.4 & 1.15 & 222 & 0.09 & 0.2 & .54 & 2.6 & 18.5 & 2.2 & - \\
CJS1.7 & 0.81 & 41 & 0.18 & 8.9 & 1.8 & 2.8 & 16.5 & 2.9 & 20 \\
CJS1.12 & 0.78 & 64 & 0.18 & 1.8 & 1.7 & 8.0 & 10.7 & 7.0 & 5.0 \\
CJS2.6 & 1.20 & 232 & 0.09 & 0 & 1.0 & 3.3 & 13.1 & 2.3 & - \\
CJS2.12 & 0.79 & 25 & 0.16 & 4.7 & 2.4 & 2.8 & 3.4 & 2.4 & - \\
CJS3.4 & 0.76 & 114 & 0.17 & 1.5 & 1.3 & 2.7 & 17.9 & 2.1 & 4.3 \\
CJS3.7 & 1.57 & 84 & 0.07 & 1.2 & 1.0 & 3.2 & 11.0 & 2.2 & - \\
CJS4.4(A) & 1.30 & 119 & 0.15 & 0.7 & .86 & 5.3 & 20.3 & 10.9 & 63 \\
CJS4.10 & 1.40 & 175 & 0.17 & 0.2 & .68 & 2.0 & 14.6 & 2.6 & 24 \\
EJS1.4 & 0.89 & 14 & 0.22 & 17 & 3.1 & 18.1 & 7.6 & 21.5 & 5.1 \\
EJS2.5 & 0.74 & 20 & 0.22 & 19 & 2.4 & 7.6 & 20.8 & 6.0 & 3.8 \\
EJS2.7 & 0.81 & 76 & 0.20 & 2.9 & 2.7 & 10.1 & 12.0 & 19.6 & 11 \\
EJS3.5 & 0.96 & 35 & 0.16 & 8.3 & 2.8 & 7.7 & 14.3 & 7.4 & 6.3 \\
EJS4.4 & 0.77 & 29 & 0.34 & 12 & 2.6 & 13.1 & 20.7 & 22.2 & 12 \\
EJS4.9 & 0.99 & 24 & 0.74 & 7.1 & 1.9 & 11.6 & 4.7 & 18.3 & 9.7
\end{tabular}

Table 1: Summary of results. Individual bodies with $M_{f}>0.7 M_{E}$ from OML06 runs are identified by xJSn.p where $\mathrm{x}$ is either $\mathrm{C}$ (circular Jupiter and Saturn) or $\mathrm{E}$ (eccentric), $\mathrm{n}$ is the run number and $\mathrm{p}$ is the particle number within the run. $M_{f}$ is the final body mass in Earth masses, $T_{i m p}$ is the timing of the final giant impact, $\gamma$ is the impactor:target mass ratio for the final giant impact, $\gamma_{v n r}$ is the fraction of the total mass added after the final giant impact. $\varepsilon_{W}$ and $f^{H f / W}$ are the values for the final body (tar.) and impactor (imp.), evaluated at the present day and calculated assuming $k=0.5$. $T_{90 \%}$ is the time taken for the last impactor to reach $90 \%$ of its final mass; a dash indicates that its mass at $t=0$ already exceeds this value. Particle A is discussed in the text and shown in Figs 3 and 4. 

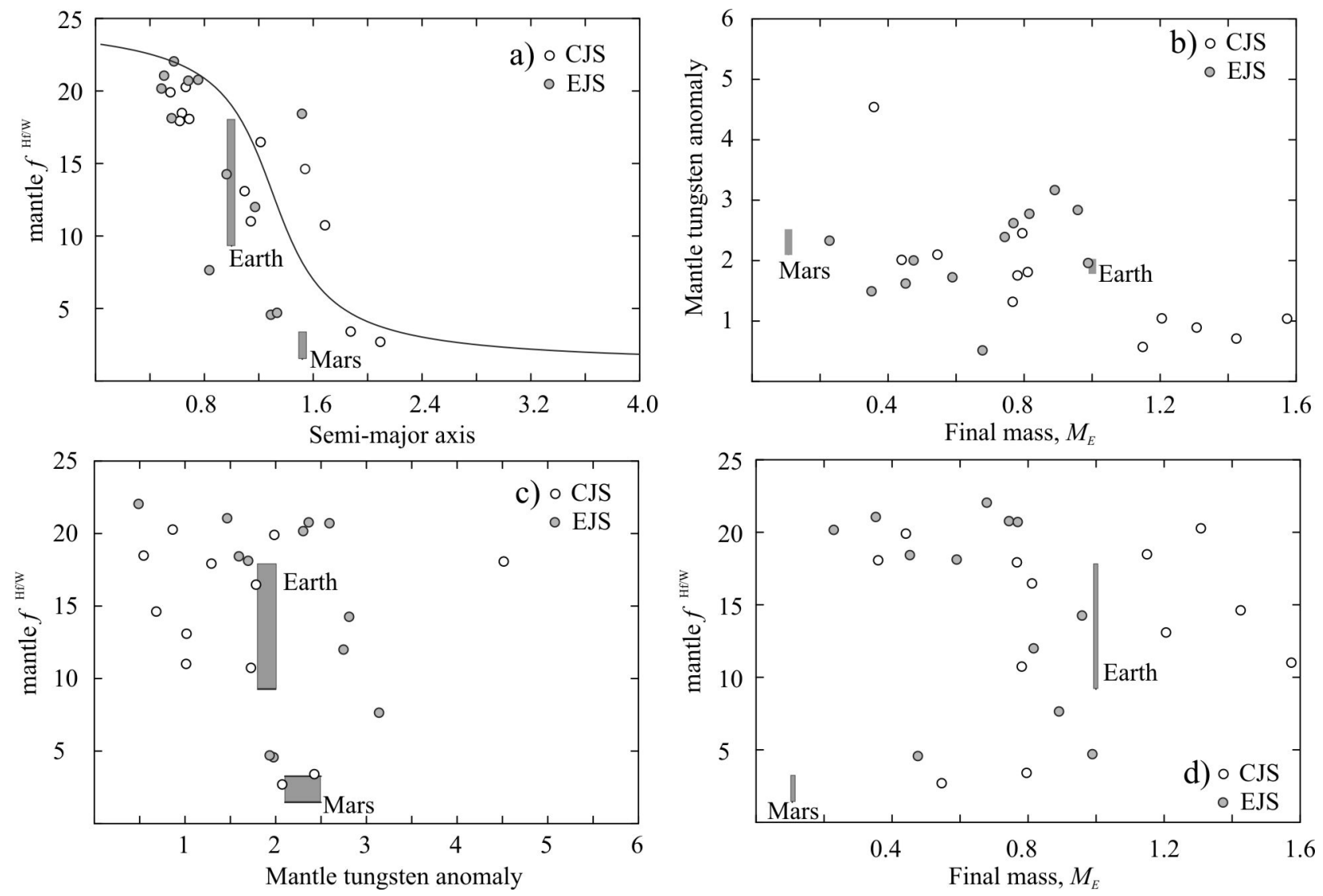

Figure 1: a) Mantle $f^{H f / W}$ of surviving bodies as a function of final semi-major axis. Here the equilibration factor $k=0.5$ and bodies greater than $0.2 M_{E}$ are plotted, where $M_{E}$ is an Earth mass. Earth and Mars (shergottite source) values (see Section 2.2) are plotted as grey rectangles. Solid line denotes initial assumed variation in $f^{H f / W}$ (see text). CJS and EJS refer to model runs with circular (C) or eccentric (E) orbits for the giant planets. b) Mantle tungsten anomaly as a function of final mass. c) Variation of mantle tungsten anomaly with mantle $f^{H f / W}$. d) Mantle $f^{H f / W}$ as a function of final body mass. 


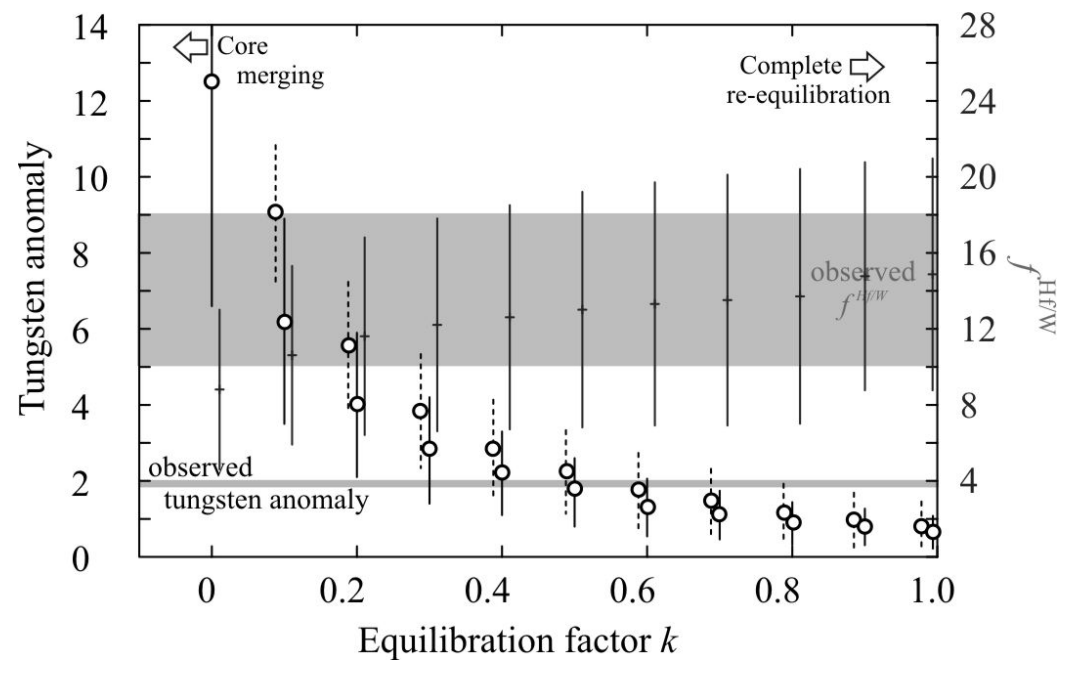

Figure 2: Circles are mean and standard deviation of mantle tungsten anomaly $\varepsilon_{W}$ as a function of equilibration factor $k$, calculated using all bodies with final masses $\geq 0.7 M_{E}$. Solid and dashed lines are using variable and constant $\left(D_{W}=29\right)$ tungsten partition coefficients, respectively. The crosses show the mean and standard deviation of $f^{H f / W}$ for the variable- $D_{W}$ case. The shaded boxes give the terrestrial values for $\varepsilon_{W}$ and $f^{H f / W}$. Plotted symbols are offset for clarity. Table 1 tabulates results for individual bodies. 

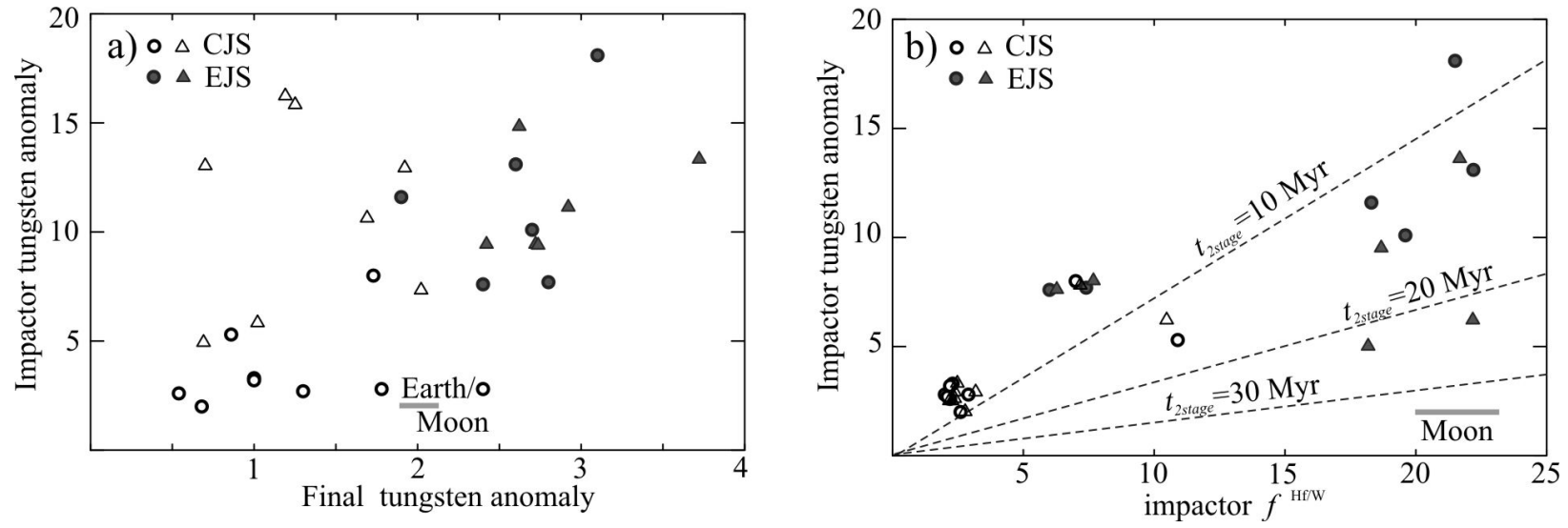

Figure 3: a) Mantle tungsten anomaly (corrected to the present-day value) of final impactors compared with the present-day mantle tungsten anomaly of the final body. Circles refer to models with spatially-variable tungsten partitioning; triangles assume $D_{W}=29$ throughout. Shaded boxes give the present-day Earth/Moon value. Here $k=0.5$. b) Variation of impactor tungsten anomaly with impactor $f^{H f / W}$. Dashed lines are expected results for two-stage differentiation at the quoted time (Jacobsen, 2005, eq. 13). Here circles refer to the nominal model results $(k=0.5$, spatially-variable partitioning) while triangles assume $k=0.3$ and all accretion timescales have been multiplied by a factor of 3 (see Section 3.3). Body A is discussed in the text; only bodies with final mass $\geq 0.7 M_{E}$ are plotted.
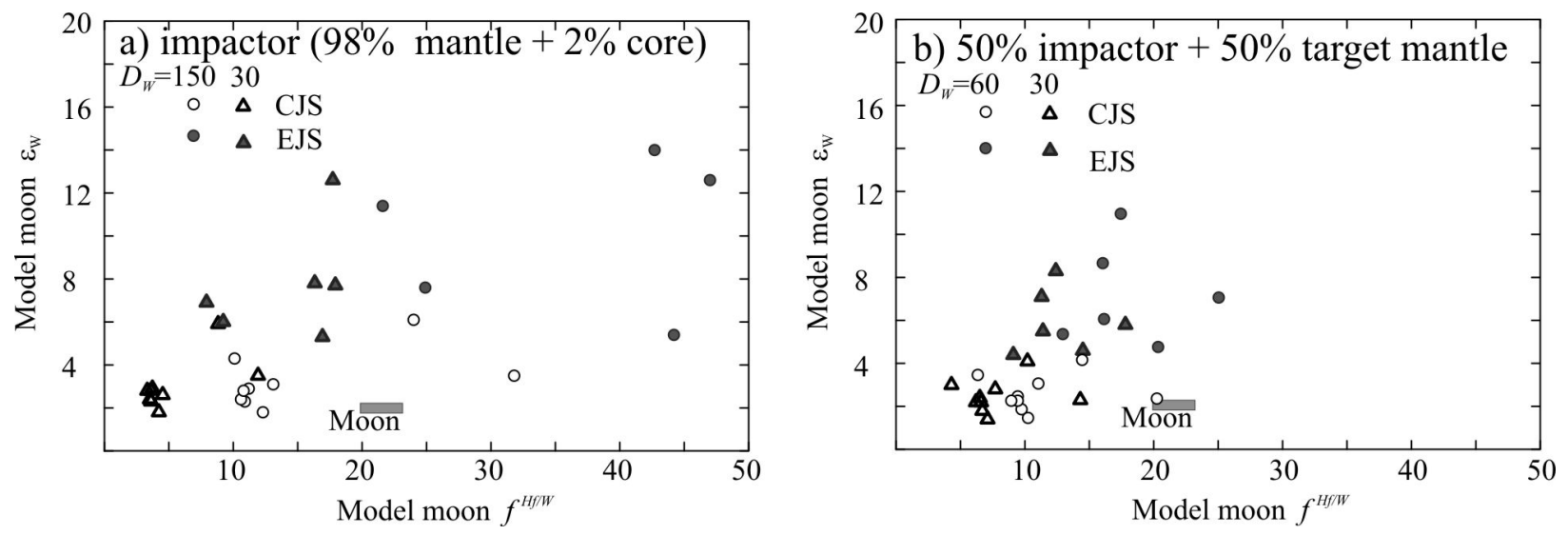

Figure 4: a) Present-day mantle tungsten anomaly and $f^{H f / W}$ for the model moon. Here $\varepsilon_{W}$ and $f^{H f / W}$ are calculated assuming that $2 \%$ of the impactor core is mixed with the impactor mantle, and then differentiation occurs based on a specified partition coefficient $D_{W}$ (=30 or 150). Observed lunar values are shown as the shaded box. b) As for a), but the impactor mix ( $2 \%$ core $+98 \%$ mantle) is then mixed with an equal mass of target mantle material, prior to differentiation $\left(D_{W}=30\right.$ or 60$)$. Body A is discussed in the text. 